\title{
A risk score model for the metastasis of level Ib lymph node based on the clinicopathological features of nasopharyngeal carcinoma in a large sample
}

\author{
WEI YI ${ }^{*}{ }^{*}$, XIAN LI $^{2 *}$, ZHIGANG LIU ${ }^{3}$, CHANGBIN JIANG $^{1}$, DAOLI NIU ${ }^{1}$ and YUNFEI XIA ${ }^{3}$ \\ Departments of ${ }^{1}$ Radiotherapy, ${ }^{2}$ Radiology, The First Affiliated Hospital of Guangzhou Medical University, \\ Guangdong 510120; ${ }^{3}$ Department of Radiotherapy, Sun Yat-Sen University Cancer Center, Guangdong 510060, P.R. China
}

Received April 11, 2014; Accepted May 5, 2014

DOI: $10.3892 / \mathrm{mco} .2014 .315$

\begin{abstract}
The aim of the present study was to develop a metastatic risk score model of neck level Ib lymph nodes in primary nasopharyngeal carcinoma (NPC) to guide the level Ib radiotherapy. There were a total of 1,557 patients enrolled in the study, and of these patients, 1,145 were included in the training set. Univariate $\chi^{2}$ analysis and multivariate logistic regression analyses were used to screen the independent risk factors to construct the risk score model. A total of 85 patients in the validating set underwent a pathology biopsy of level Ib lymph nodes to test the model. The remaining 327 patients from the prognostic-research set were used to evaluate the prognostic impact of level Ib irradiation in high- and low-risk groups. The independent risk factors in the model were carotid sheath involvement, the maximal diameter of the neck lymph nodes $(\geq 20 \mathrm{~mm})$ and the involvement of the level II/III/IV lymph nodes. The involvement of level IV was assigned score 2 and the other risk factors were assigned score 1. According to the total scores, the patients were divided into the low(total score, 0-1; level $\mathrm{Ib}$ metastasis rate, $0.5 \%$ ) and high-risk groups (total score, 2-4; level Ib metastasis rate, $8.5 \%$ ). In the validating set, the metastatic rate of level Ib in 43 low-risk patients was $0 \%$, and the rate was $31.0 \%$ (13/42) in 42 high-risk patients. In the prognostic-research set, the prognosis of
\end{abstract}

Correspondence to: Dr Yunfei Xia, Department of Radiotherapy, Sun Yat-Sen University Cancer Center, 651 Dongfeng East Road, Guangdong 510060, PR. China

E-mail: yunfeixia@yahoo.com

Dr Daoli Niu, Department of Radiotherapy, The First Affiliated Hospital of Guangzhou Medical University, 151 YanJiang Road, Guangzhou, Guangdong 510120, P.R. China

E-mail: daoli_niu@163.com

*Contributed equally

Key words: nasopharyngeal carcinoma, radiotherapy, level Ib lymph node, lymph node metastasis, risk score model, locoregional recurrence
137 low-risk patients was not affected by level Ib irradiation. However, level Ib unirradiation was an independent prognostic factor for the locoregional recurrence in 190 high-risk patients. According to the data, the novel score model could help assess the metastatic risk of level Ib in primary NPC, and the radiotherapy on level Ib may impact the locoregional recurrence in high-risk patients.

\section{Introduction:}

Nasopharyngeal carcinoma (NPC) has a unique racial and geographical epidemiology, with the highest rate of occurrence in Guangdong (southern China). The majority of NPC cases that occur in this high-risk area are poorly-differentiated and prone to infiltrative growth and metastasis. The metastatic incidence of neck lymph node may be as high as $78.9 \%$ (1).

Radiotherapy is indispensable for comprehensive NPC treatment. With the development of radiotherapy techniques, the 5-year overall survival (OS) rate of NPC has increased from $40 \%$ in the 1960 s (2) to $75 \%$ by the end of the 1990 s (3), and to $>80 \%$ due to the introduction of intensity-modulation radiation therapy (IMRT) (4). As survival rates have increased, the concern of the late complications due to radiotherapy have also increased. The clinical target volume (CTV) of the lymph node neck levels is one critical area for reducing the side-effects of radiotherapy. The most problematic one is the CTV of the level Ib lymph node, which mainly comprises the submandibular lymph node surrounding the submandibular gland (SMG) (5).

The metastatic incidence of the level Ib lymph node ranges from 2.0-8.5\% according to image-based analysis (6-8). During the $2 \mathrm{D}$ radiotherapy era, the entire region of level $\mathrm{Ib}$ was included in the CTV. As the irradiation of the level Ib lymph node can significantly increase the dose absorbed by the surrounding normal tissues, the function of SMG could be extremely damaged. Damage to the salivary glands results in xerostomia in $>90 \%$ patients (9). The study by Kuten et al (10) reported that a reduced dose to the SMG could effectively alleviate xerostomia. Certain surgeons have developed SMG-transfer surgery to preserve the SMG and this procedure achieves good results $(11,12)$, but the invasiveness limits its use in clinical practice. In the IMRT era, numerous 
studies identified the importance of SMG protection as it was observed that xerostomia could not be sufficiently reduced when the parotid gland was well preserved and the SMG was not (13-16). In principle, determining the target volume for the level $\mathrm{Ib}$ lymph node is problematic. Due to the low-metastatic rate of level Ib, selective irradiation may be reasonable. Specific indications for the irradiation of level Ib lymph nodes have been formulated $(17,18)$, but the clinical evidence is not convincing.

Therefore, the present study was performed to provide certain evidence-based indications for the irradiation of level Ib lymph node in NPC. First, the independent risk factors for the metastasis of level Ib lymph node were identified based on the analysis of the magnetic resonance imaging (MRI)/computed tomography (CT) images from a large sample of 1,145 cases in the training set. Subsequently, a score model was built according to those independent risk factors. The model was used to separate the patients into lowand high-risk groups. In the validating set, 43 low-risk and 42 high-risk patients underwent a pathological biopsy of the level Ib lymph nodes to validate the accuracy of the model. A further 327 patients with a long-term outcome were included in the prognosis-research set. The prognostic effects of level Ib lymph node irradiation were assessed in low- and high-risk patients.

\section{Patients and methods}

Patients. The study enrolled 1,557 patients with primary NPC. All the patients were diagnosed and initially treated at the Sun Yat-Sen University Cancer Center or the First Affiliated Hospital of Guangzhou Medical University (Guangdong, China). All the patients underwent CT/MRI scans of the nasopharynx prior to radiotherapy.

Of those patients, 1,145 cases were included in the training set. The training set comprised of two patient groups. One group of 933 patients was randomly selected from 6,519 hospitalized patients at the Sun Yat-Sen University Cancer Center between January 1990 and December 1999. The 212 patients of the second group were from a clinical trial by the National High Technology Research and Development Program 863 (2006AA02Z4B4) and treated by Dr Y.F. Xia between January 2004 and April 2010.

A total of 85 patients were included in the validation set. All the patients consented to a biopsy in level Ib lymph nodes prior to radiotherapy. The pathological biopsy of the level Ib lymph node was obtained between April 2003 and February 2011 in the Sun Yat-Sen University Cancer Center or The First Affiliated Hospital of Guangzhou Medical University.

In the prognostic-research set, all the patients who underwent 3D radiotherapy between November 2001 and November 2004 in the Sun Yat-Sen University Cancer Center were investigated. There were 335 patients who had complete follow-up data and no distance metastasis prior to the treatment. In the practical work, the level Ib lymph nodes that fulfilled the radiographic standard of metastasis were recognized as requiring irradiation. However, the small lymph nodes that did not fulfill the standard were of more concern. Therefore, eight patients were excluded, as they fulfilled the radiographic standard of level $\mathrm{Ib}$ lymph nodes metastasis.
There were 327 patients remaining in the prognostic-research set. No overlap existed among the training, validation and prognostic-research sets.

Tumor-node-metastasis stage. The tumors were staged using the 7th edition of the Union for International Cancer Control/American Joint Commission on Cancer stage manual in all the patients (19).

Metastatic criteria of the level Ib lymph node. The radiographic diagnostic criteria for the metastasis of the level $\mathrm{Ib}$ lymph node followed the standard of van den Brekel et al (20), as: i) Lymph nodes with a minimal axial diameter $>10 \mathrm{~mm}$; ii) groups of three or more borderline lymph nodes with a minimal axial diameter of $8 \mathrm{~mm}$; and iii) nodes with a central necrosis or a rim of enhancement.

Biopsy of the level Ib lymph node. The methods for the biopsy of level Ib lymph nodes included ultrasound-guided core and excisional biopsies. Excisional biopsy was used in 39 patients who were willing to undergo SMG-transfer surgery prior to radiotherapy. All the level Ib lymph nodes of the 39 patients were removed for pathological examination. Ultrasound-guided core biopsy was used in 46 patients. In those patients, at least one level Ib lymph node (diameter $>6 \mathrm{~mm}$ ) was examined. No less than two tissue specimens were obtained in each examined lymph node.

Treatment. All the patients were irradiated using an accelerator producing 6-8 MV X-rays. The primary tumor was irradiated with a dose of 66-70 Gy. Additional radiotherapy boosts of 8-12 Gy were administered following the delivery of the standard dose for residual tumors or if the skull base was involved. The neck received 50-70 Gy, depending on the lymph node involvement. The radiotherapy techniques consisted of opposing lateral faciocervical fields to cover the nasopharynx and upper cervical lymphatic drainage region, with one low anterior cervical field to cover the lower cervical region. Following 36-40 Gy, opposing lateral preauricular fields were used for the primary tumor, and anterior split-neck fields were used for the cervical region. When the nasal cavity was involved, a supplementary anterior facial field was added.

Irradiation of level Ib lymph node. In the prognostic-research set, 327 patients underwent 3D radiotherapy. The CTV2 of 180 patients covered all the visible lymph nodes of level $\mathrm{Ib}$ (the front line included prevascular lymph nodes). The prescribed dose of CTV2 was $50 \mathrm{~Gy}$. The CTV2 of another 147 patients did not cover all the visible level Ib lymph nodes (Fig. 1).

Follow-up. The patients were followed up by phone calls, letters or outpatient reviews at least once a year. The follow-up time was calculated from the start date of radiotherapy and ceased on 31 December 2012.

Statistical analysis. Statistical analysis was performed using the SPSS 16.0 package (SPCC, Inc., Chicago, IL, USA). In the training set, the occurrence of level Ib lymph nodes metastasis as a binary outcome was analyzed by multivariate binary logistic regression, considering 10 explanatory variables. 


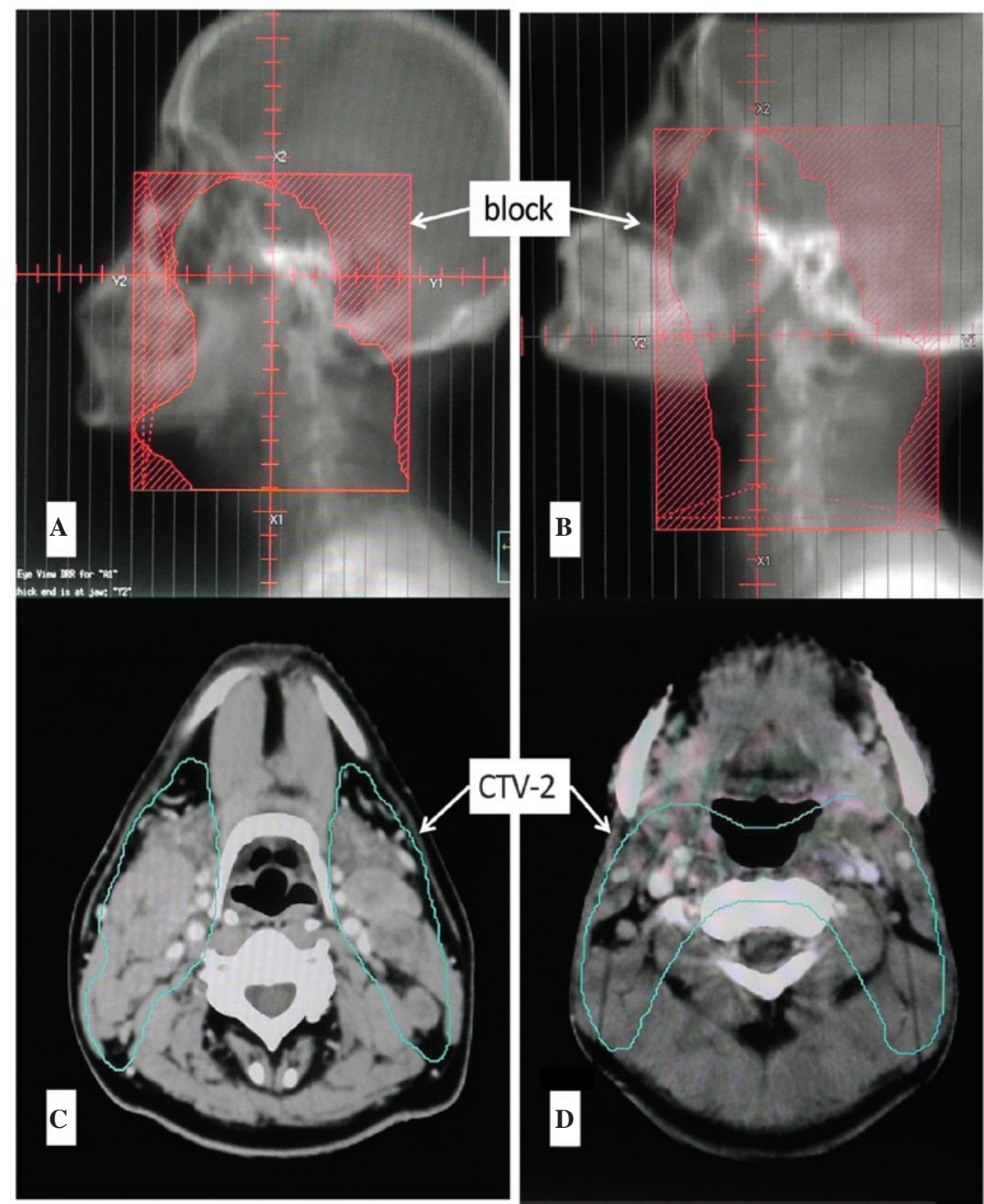

Level Ib irradiated

Level Ib unirradiated

Figure 1. Schematic diagrams of level Ib irradiation. DRR of a (A) level Ib irradiated patient and a (B) level Ib unirradiated patient. The clinical target volume (CTV) contouring of a (C) level Ib irradiated patient and a (D) level Ib unirradiated patient.

The multivariated logistic regression included the factors associated with level Ib lymph node metastasis, either by significance in univariate analysis or by clinical experience. A reduced model was obtained by a backward stepwise selection procedure with the stay criterion set at 0.10 . The part and partial correlations and collinearity diagnostics were used to test for collinearity. The tolerance $<0.1$, or variance inflation factor $>2$, were considered problematic. The adequacy of the logistic regression models was assessed using the Hosmer and Lemeshow goodness-of-fit test. The regression coefficient of each independent variable was subsequently modified into an integer numerical value to simplify the computation (21).

In the prognostic-research set, the Kaplan-Meier plots and the log-rank test were used to estimate the OS, the locoregional recurrence rate (LRR) and the distant metastatic rate (DMR) for all the 327 patients. The multivariable Cox proportional-hazards model analysis was conducted to determine whether the association between level Ib irradiation and post-treatment outcomes was independent of other known prognostic factors. The age, gender, chemotherapy, T stage and $\mathrm{N}$ stage were assessed and entered in a backward stepwise Cox proportional-hazards model to identify the predictors of the clinical outcomes. The level of significance chosen was 0.05. The hazard ratios in the prognostic research were used to calculate the relative risk of recurrence or distance metastasis. Those calculations were based on the Pike estimate, using the observed and expected numbers of events, as calculated in the log-rank test statistic; $95 \%$ confidence intervals used variances derived from the information matrix.

The proportions were compared using Fisher's exact test and $\mathrm{P}<0.05$ was considered to indicate a statistically significant difference. All the reported P-values were two-sided. 


\section{Results}

Clinicopathological characteristics. The characteristics of the 1,145 patients in the training set and 85 patients in the validating set are described in Table I.

\section{Construction of score model in the training set}

Identification for metastatic risk factors of level Ib lymph node. In the training set, the metastatic rate of the level $\mathrm{Ib}$ lymph node was $5.9 \%(67 / 1,145)$. Univariate and multivariate analyses were used to identify the association between the clinical factors and level Ib metastasis (Table II). In total, 10 explanatory variables were considered. The multivariate logistic regression included the factors associated with level Ib lymph node metastasis either by significance in univariate analysis or by clinical experience. Those factors included: Age, gender, pathology [type World Health Organization (WHO) I vs. type WHO II/III], T stage, N stage, nasal cavity involvement, maxillary sinus involvement, carotid sheath involvement, neck lymph node level involvement (uninvolved vs. level II/III involved vs. level IV involved) and maximum neck lymph node diameter of $>20 \mathrm{vs}$. $<20 \mathrm{~mm}$. The age was dichotomized at the median value of 45 years. The test for collinearity indicated that $\mathrm{N}$ stage was problematic, and therefore it was excluded. The multivariated logistic regression indicated that the carotid sheath involvement, maximal diameter of the neck lymph node $(\geq 20 \mathrm{~mm})$ and involvement of level II/III/IV lymph nodes were three independent risk factors that were significantly associated with the metastasis of level Ib lymph node.

Risk score model for the metastasis of level Ib lymph node. To create a systemic and feasible score model in a clinical practice, an integral score derived from the $n$ value for each risk factor was assigned. The $n$ value was calculated by the regression coefficients of each independent risk factor: $\mathrm{n}=1 \mathrm{n}$ odds ratio (OR) (21). When the risk factor was absent, a score of 0 was recorded, and 1 was recorded when there was carotid sheath involvement, maximal diameter of neck lymph node ( $\geq 20 \mathrm{~mm}$ ) or involvement of level II/III lymph nodes. If a level IV lymph node was involved, a score of 2 was recorded (Table III). The total scores were calculated by collating all the scores of all three risk factors. This indicated that patients with total scores of $0-1$ had a low-metastatic rate of level Ib lymph node $(0.5 \%)$. However, the patients with total scores of 2-4 had a much higher metastatic rate of level $\mathrm{Ib}$ lymph node $(8.5 \%)$. Therefore, the patients with total scores of $0-1$ were identified as low-risk patients, and the patients with total scores of 2-4 were identified as high-risk patients. The statistical difference of the level Ib metastatic rate between the two groups was significant $(\mathrm{P}<0.001)$ (Table III).

Test of score model in the validating set. The level Ib lymph nodes in 85 patients were pathologically examined. According to the score system established by the aforementioned training set, 43 cases were classified as low-risk and 42 cases as high-risk patients. Notably, the score model accurately predicted the observed outcomes in the pathological validating set. A total of 13 of the 85 patients had the metastasis of level Ib lymph node. There was no metastasis of the level Ib lymph node in the low-risk patients. However, in the high-risk patients, there
Table I. Clinicopathological characteristics in the training and validating sets.

\begin{tabular}{|c|c|c|c|c|}
\hline \multirow[b]{2}{*}{ Characteristic } & \multicolumn{2}{|c|}{ Training set } & \multicolumn{2}{|c|}{ Validating set } \\
\hline & $\mathrm{n}=1145$ & $\%$ & $\mathrm{n}=85$ & $\%$ \\
\hline \multicolumn{5}{|l|}{ Age } \\
\hline$\leq 45$ years & 590 & 51.5 & 48 & 56.5 \\
\hline$>45$ years & 555 & 48.5 & 37 & 43.5 \\
\hline \multicolumn{5}{|l|}{ Gender } \\
\hline Male & 864 & 75.5 & 65 & 76.5 \\
\hline Female & 281 & 24.5 & 20 & 23.5 \\
\hline \multicolumn{5}{|l|}{ Histology } \\
\hline WHO I & 10 & 0.9 & 1 & 1.2 \\
\hline WHO II/III & 1135 & 99.1 & 84 & 98.8 \\
\hline \multicolumn{5}{|c|}{ Clinical stage (UICC) } \\
\hline I & 82 & 7.2 & 4 & 4.7 \\
\hline II & 437 & 38.2 & 22 & 25.9 \\
\hline III & 362 & 31.6 & 33 & 38.8 \\
\hline IV & 264 & 23.1 & 26 & 30.6 \\
\hline \multicolumn{5}{|l|}{ T classification } \\
\hline $\mathrm{T} 1$ & 251 & 21.9 & 8 & 9.4 \\
\hline $\mathrm{T} 2$ & 466 & 40.7 & 26 & 30.6 \\
\hline $\mathrm{T} 3$ & 227 & 19.8 & 34 & 40.0 \\
\hline $\mathrm{T} 4$ & 201 & 17.6 & 17 & 20.0 \\
\hline \multicolumn{5}{|l|}{$\mathrm{N}$ classification } \\
\hline N0 & 317 & 27.7 & 20 & 23.5 \\
\hline N1 & 533 & 46.6 & 26 & 30.6 \\
\hline $\mathrm{N} 2$ & 207 & 18.1 & 26 & 30.6 \\
\hline N3 & 88 & 7.7 & 13 & 15.3 \\
\hline
\end{tabular}

WHO, World Health Organization; UICC, Union for International Cancer Control.

were 13 cases of level Ib lymph nodes metastasis. The metastatic rate of level Ib lymph node was as high as $31 \%$. The statistical difference between the low- and high-risk patients was significant.

Prognostic research. The prognostic effect of level Ib irradiation in low- and high-risk patients was evaluated in another group of 327 cases. Those patients were followed-up for a mean of 58 months (range, 4-93 months). Their clinical characteristics are shown in Table IV.

When the 327 patients were analyzed collectively, the level Ib irradiation did not influence the OS, LRR and DMR significantly (data not shown). Subsequently, further analysis was conducted. Those patients were separated into low- (137 cases) and high-risk patients (190 cases) according to the aforementioned risk-score model. In the low-risk patients, level Ib irradiation still had no significant influence on OS, LRR or DMR (Fig. 2A, C and E). In the high-risk patients, unadjusted survival analysis revealed that LRR was significantly low in level Ib-irradiated patients (Fig. 2D). The 
Table II. Univariate and multivariate analysis for the clinical predictors of level Ib metastasis.

\begin{tabular}{|c|c|c|c|c|c|c|}
\hline \multirow[b]{2}{*}{ Characteristic } & \multicolumn{3}{|c|}{ Univariate analysis } & \multicolumn{3}{|c|}{ Multivariate analysis } \\
\hline & $\begin{array}{c}\text { Level Ib } \\
\text { metastasis (-) }\end{array}$ & $\begin{array}{c}\text { Level Ib } \\
\text { metastasis (+) }\end{array}$ & P-value & $\begin{array}{l}\text { Hazard } \\
\text { ratio }\end{array}$ & $\begin{array}{l}95 \% \text { confidence } \\
\text { interval }\end{array}$ & P-value \\
\hline \multicolumn{7}{|l|}{ Age } \\
\hline$\leq 45$ years & 557 & 33 & 0.708 & - & - & - \\
\hline$>45$ years & 521 & 34 & & & & \\
\hline \multicolumn{7}{|l|}{ Gender } \\
\hline Male & 809 & 55 & 0.241 & 0.630 & $0.322-1.232$ & 0.177 \\
\hline Female & 269 & 12 & & & & \\
\hline \multicolumn{7}{|l|}{ Histology } \\
\hline WHO I & 10 & 0 & 1.000 & - & - & - \\
\hline WHO II/III & 1068 & 67 & & & & \\
\hline \multicolumn{7}{|l|}{ T stage } \\
\hline $\mathrm{T} 1$ & 240 & 11 & 0.240 & 0.813 & $0.580-1.139$ & 0.229 \\
\hline $\mathrm{T} 2$ & 436 & 30 & & & & \\
\hline T3 & 209 & 18 & & & & \\
\hline $\mathrm{T} 4$ & 193 & 8 & & & & \\
\hline \multicolumn{7}{|l|}{$\mathrm{N}$ stage } \\
\hline N0 & 317 & 0 & $<0.001^{\mathrm{a}}$ & - & - & - \\
\hline N1 & 506 & 27 & & & & \\
\hline $\mathrm{N} 2$ & 184 & 23 & & & & \\
\hline N3 & 71 & 17 & & & & \\
\hline \multicolumn{7}{|c|}{ Nasal cavity involvement } \\
\hline No & 866 & 52 & 0.635 & 1.288 & $0.665-2.495$ & 0.453 \\
\hline Yes & 212 & 15 & & & & \\
\hline \multicolumn{7}{|c|}{ Maxillary sinus involvement } \\
\hline No & 1033 & 45 & 0.515 & 0.420 & $0.049-3.623$ & 0.430 \\
\hline Yes & 66 & 1 & & & & \\
\hline \multicolumn{7}{|c|}{ Carotid sheath involvement } \\
\hline No & 544 & 20 & $0.001^{\mathrm{a}}$ & 2.106 & $1.204-3.682$ & $0.009^{\mathrm{a}}$ \\
\hline Yes & 534 & 47 & & & & \\
\hline \multicolumn{7}{|c|}{ Neck lymph node level involvement } \\
\hline No & 327 & 0 & $<0.001^{\mathrm{a}}$ & 6.965 & 3.866-12.548 & $<0.001^{\mathrm{a}}$ \\
\hline Level II/III & 708 & 49 & & & & \\
\hline Level IV & 43 & 18 & & & & \\
\hline \multicolumn{7}{|c|}{ Maximum neck lymph node diameter } \\
\hline$<20 \mathrm{~mm}$ & 449 & 6 & $<0.001^{\mathrm{a}}$ & 2.212 & 0.907-5.394 & $0.081^{\mathrm{a}}$ \\
\hline$\geq 20 \mathrm{~mm}$ & 629 & 61 & & & & \\
\hline
\end{tabular}

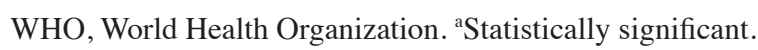

5-year LRR in unirradiated patients was $14.3 \%$, however, in level Ib-irradiated patients it was only $4.4 \%$. Multivariate survival analysis included variants of $\mathrm{T}$ stage, $\mathrm{N}$ stage, chemotherapy, gender and age, which indicated that level Ib irradiation was the independent prognostic factor for LRR in the high-risk group (Table V). However, level Ib irradiation had no significance influence on OS and DMR in the high-risk group (Fig. 2B and F).

\section{Discussion}

Based on the comprehensive analysis of the large sample, the carotid sheath involvement, maximal diameter of neck lymph node and involvement of neck level II/III/IV lymph node were identified as the credible metastatic risk factors for level $\mathrm{Ib}$ lymph nodes in primary NPC. These risk factors could be interpreted by lymphotomy. 
In general, the metastasis of lymph nodes in NPC is characterized by systematic spreading down the neck along the deep cervical lymph nodes $(6,22)$. The level Ib lymph nodes are superficial cervical lymph nodes and they drain the lymph from the floor of the mouth, buccal mucosa, gingiva, face and anterior region of the orbit and nasal cavity to the deep cervical lymph nodes. Therefore, the tumor cells of NPC could not reach the level Ib lymph nodes by direct lymph drainage. Lymphatic reflux caused by blockage of the deep cervical lymph nodes could be the possible reason for level Ib lymph nodes metastasis.

The first risk factor of carotid sheath involvement is caused by the primary NPC invasion or metastasis of the retropharyngeal nodes, which are usually difficult to identify by CT images. The retropharyngeal lymph nodes are the first echo node of the NPC $(6,22)$, which is regarded as an indicator of neck metastasis. Certain studies have indicated that carotid sheath involvement is the transfer station for neck metastasis (23). In the present study, metastasis of the level Ib lymph node was rarely found without carotid sheath involvement. The second risk factor, maximum diameter of the neck lymph nodes, was also significantly associated with level Ib lymph nodes metastasis. The patients with $\leq 20 \mathrm{~mm}$ maximum diameter of neck lymph nodes had the lower level Ib metastatic rate (only 1.3\%). When the maximum diameter of the neck lymph nodes was 20-39 $\mathrm{mm}$, the rate quickly reached $8.5 \%$. However, the level $\mathrm{Ib}$ metastatic rate moderately reached $9.4 \%$ when the maximum diameter of neck lymph nodes was $>40 \mathrm{~mm}$. This indicated that the enlarged lymph nodes could block the lymph drainage and generate reflux efficiently in the $20 \mathrm{~mm}$ maximum diameter of those metastatic lymph nodes. However, further enlargement of the lymph nodes could not significantly increase the reflux. The third risk factor, involvement of neck level II/III/IV lymph nodes, had a greater role. This risk factor was closely and positively associated with metastasis of the level Ib lymph nodes (24). When the level IV lymph node was involved, the metastatic incidence of the level Ib lymph node $(18 / 61,29.5 \%)$ was almost five times higher than the general average $(61 / 1,145,5.3 \%)$. According to a comprehensive analysis of the aforementioned results, when the carotid sheath was involved or the neck lymph nodes were $\geq 20 \mathrm{~mm}$, the reflux to level Ib may occur. Additionally, the involved extension of the neck levels was the most important risk factor for level $\mathrm{Ib}$ lymph nodes metastasis.

Other various factors, including nasal cavity and maxillary sinus involvement, were previously considered to be associated with level Ib metastasis. However, the present study could not support those assumptions. For nasal cavity involvement, it is possible that the tumor could spread to the level Ib lymph nodes following the lymphatic drainage from the anterior one-third of the nasal cavity. However, this situation was rarely clinically observed as almost all the patients had been treated prior to the occurrence. The maxillary sinus involvement was also observed, but not associated with the metastasis of level Ib lymph nodes as the lymph of the maxillary sinus drained to the nasopharynx without direct linkage with the level Ib lymph node.

In the validation process, certain significant identifications were of note. By comparing the pathological and radiographic results of 85 patients, two noteworthy indications were found.
Table III. Calculation of the risk score and risk group.

\begin{tabular}{lcc}
\hline Variable & $\mathrm{n}=1 \mathrm{n}$ OR & Score \\
\hline Neck lymph node level involvement & & \\
$\quad$ No & 1.940 & 0 \\
Level II/III & & 1 \\
Level IV & & 2 \\
Maximum neck lymph node diameter & 0.794 & 0 \\
$\quad<20$ mm & & 1 \\
$\geq 20$ mm & & \\
Carotid sheath involvement & 0.745 & 0 \\
No & & 1 \\
Yes & Level Ib & \\
\hline & metastatic & Total \\
& rate, $\%$ & score \\
Risk group & 0.5 & $0-1$ \\
\hline Low, $\mathrm{n}=381$ & 8.5 & $2-4$ \\
High, $\mathrm{n}=764$ & & \\
\hline
\end{tabular}

OR, odds ratio.

In the low-risk group, the radiographic and pathological diagnoses were consistent in 41 out of 43 patients. In the high-risk group, the CT/MR imaging only diagnosed 4 cases of level $\mathrm{Ib}$ lymph node metastases in all 13 patients who were proved by the pathological result. These results showed that the imagine diagnosis of the level Ib lymph node metastasis was more credible in the low-risk patients. This phenomenon may be generated by two factors. Firstly, level Ib lymph nodes are the superficial lymph node, and they drain lymphatic fluid from the oral and maxillofacial, which are easy to inflame. Therefore, the level Ib lymph nodes are often reactively enlarged due to the inflammation. If the inflammatory lymph node is large enough to fulfill the diagnostic standard in the CT/MR image, a false-positive diagnosis occurs. Secondly, the metastasis of the lymph node is a continuous process that begins with a single or a cluster of tumor cells migrating into the lymph nodes, which may take months to grow large enough to be detected by CT or MR (25). This second situation causes a false-negative diagnose that usually occurs in high-risk patients.

Subsequent to the construction and validation of the risk model, it was assumed that: i) There was an extremely low risk to omit irradiation in level $\mathrm{Ib}$ in low-risk patients due to the small chance of metastasis; and ii) level Ib lymph node should be irradiated in high-risk patients due to the relatively high possibility of metastasis. Further studies on the prognosis could also support this assumption. In the low-risk group, level $\mathrm{Ib}$ irradiation had no significant effect on the prognosis. In the high-risk group, level Ib unirradiation was an independent prognostic factor of LRR.

In fact, the recurrence of the level Ib lymph nodes may be the best indicator for evaluating the risk of level Ib irradiation. In the present study, recurrences of the level Ib lymph nodes were observed in five patients (pathological diagnosis or 
Table IV. Clinical characteristics of the 327 patients for the prognostic research.

\begin{tabular}{|c|c|c|c|c|c|c|}
\hline \multirow[b]{2}{*}{ Characteristic } & \multicolumn{2}{|c|}{ Low-risk patients, $\mathrm{n}=137$} & \multirow[b]{2}{*}{ P-value } & \multicolumn{2}{|c|}{ High-risk patients, $n=190$} & \multirow[b]{2}{*}{ P-value } \\
\hline & $\begin{array}{c}\text { Level Ib } \\
\text { unirradiated }\end{array}$ & $\begin{array}{l}\text { Level Ib } \\
\text { irradiated }\end{array}$ & & $\begin{array}{c}\text { Level Ib } \\
\text { unirradiated }\end{array}$ & $\begin{array}{l}\text { Level Ib } \\
\text { irradiated }\end{array}$ & \\
\hline \multicolumn{7}{|l|}{ Age } \\
\hline$\leq 45$ & 38 & 31 & 0.395 & 40 & 54 & 0.658 \\
\hline$>45$ & 32 & 36 & & 37 & 59 & \\
\hline \multicolumn{7}{|l|}{ Gender } \\
\hline Male & 46 & 48 & 0.468 & 54 & 78 & 1.000 \\
\hline Female & 24 & 19 & & 23 & 35 & \\
\hline \multicolumn{7}{|l|}{ Clinical stage } \\
\hline I & 17 & 6 & 0.091 & 0 & 0 & 0.105 \\
\hline II & 24 & 28 & & 23 & 21 & \\
\hline III & 18 & 17 & & 30 & 60 & \\
\hline IV & 11 & 16 & & 24 & 32 & \\
\hline \multicolumn{7}{|l|}{ T classification } \\
\hline $\mathrm{T} 1$ & 18 & 10 & 0.349 & 7 & 11 & 0.176 \\
\hline $\mathrm{T} 2$ & 24 & 26 & & 36 & 36 & \\
\hline T3 & 17 & 15 & & 19 & 42 & \\
\hline $\mathrm{T} 4$ & 11 & 16 & & 15 & 24 & \\
\hline \multicolumn{7}{|l|}{$\mathrm{N}$ classification } \\
\hline No & 53 & 41 & 0.101 & 0 & 0 & 0.028 \\
\hline N1 & 15 & 19 & & 43 & 51 & \\
\hline $\mathrm{N} 2$ & 2 & 7 & & 23 & 54 & \\
\hline N3 & 0 & 0 & & 11 & 8 & \\
\hline \multicolumn{7}{|l|}{ Chemotherapy } \\
\hline No & 47 & 44 & 0.859 & 19 & 39 & 0.199 \\
\hline Yes & 23 & 23 & & 58 & 74 & \\
\hline
\end{tabular}

Table V. Multivariate prognostic analysis of the 190 high-risk patients in LRR and DMR.

\begin{tabular}{llccc}
\hline & \multicolumn{2}{c}{ LRR } & & DMR \\
\cline { 2 - 3 } Prognostic factor & Relative risk $(95 \% \mathrm{CI})$ & P-value & Relative risk (95\% CI) & P-value \\
\hline Age & $0.816(0.303-2.196)$ & 0.686 & $1.112(0.481-2.569)$ & 0.804 \\
Gender & $0.711(0.229-2.210)$ & 0.556 & $0.711(0.229-2.210)$ & 0.556 \\
Chemotherapy & $1.260(0.396-4.006)$ & 0.696 & $1.525(0.556-4.182)$ & 0.412 \\
T stage & $0.926(0.539-1.591)$ & 0.781 & $0.940(0.604-1.463)$ & 0.782 \\
N stage & $1.029(0.466-2.273)$ & 0.944 & $2.332(1.328-4.095)$ & 0.875 \\
Level Ib irradiation & $0.291(0.101-0.837)$ & 0.022 & $1.066(0.478-2.378)$
\end{tabular}

LRR, locoregional recurrence rate; DMR, distant metastatic rate; CI, confidence interval.

minimal diameter $>10 \mathrm{~mm}$ ) of the 327 prognostic-research set patients. However, four out of five cases were accompanied by locoregional recurrence, possibly due to the tumor spreading to the level Ib lymph nodes following nasopharynx or neck recurrence. Only one case of level Ib lymph nodes metastasis occurred without locoregional recurrence in a high-risk patient without level Ib irradiation. This illustrates the importance of level Ib irradiation in high-risk patients. However, the study had particular limitations. First, the sample size of the validation set was not large enough. Second, the study was entirely based on clinical factors, which limited the ability to define the high-risk patients more precisely. 


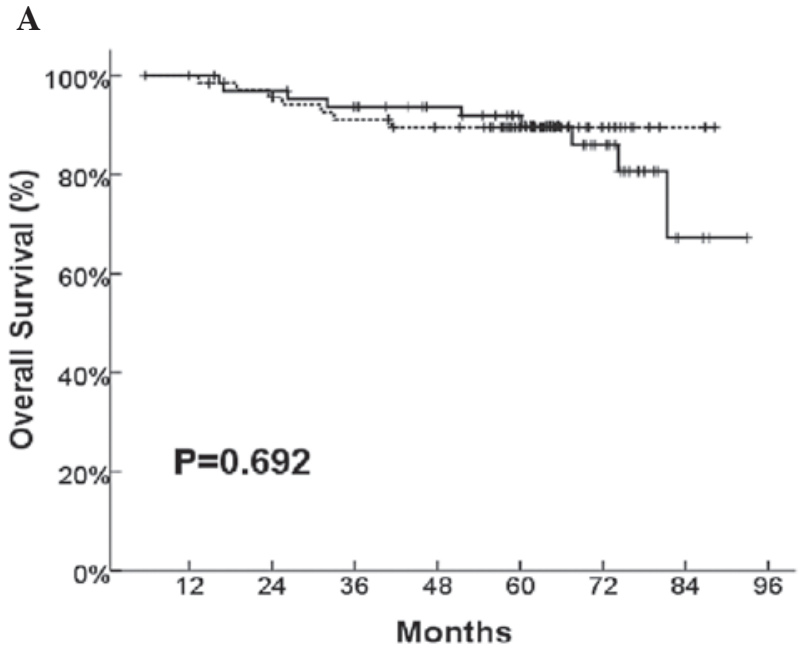

C

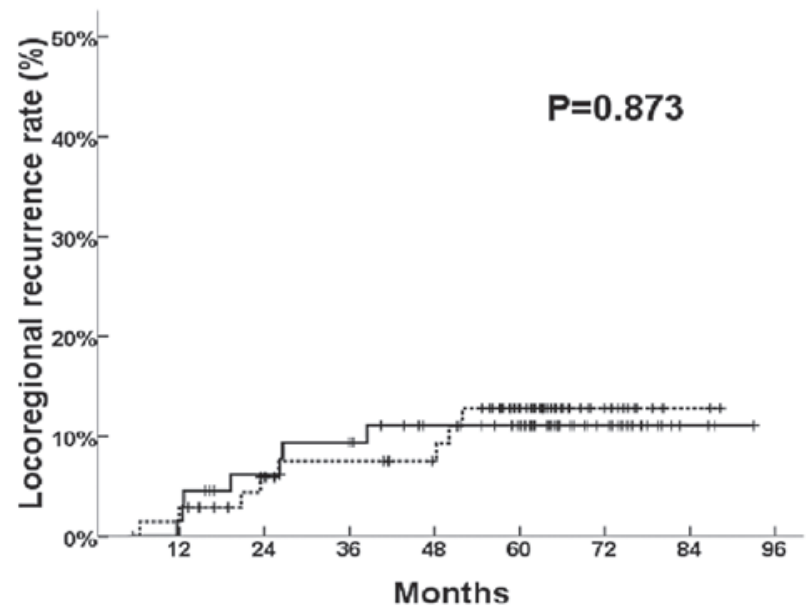

E

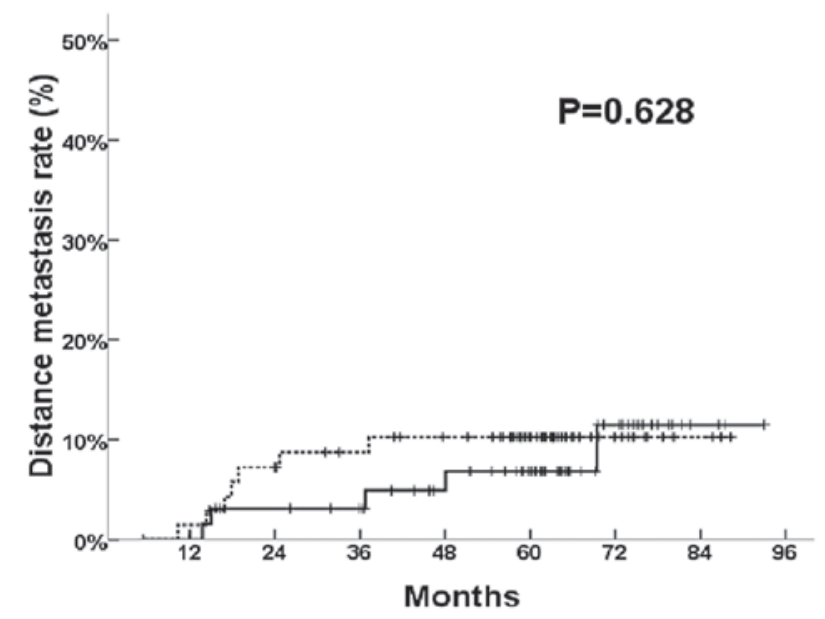

low-risk group
B

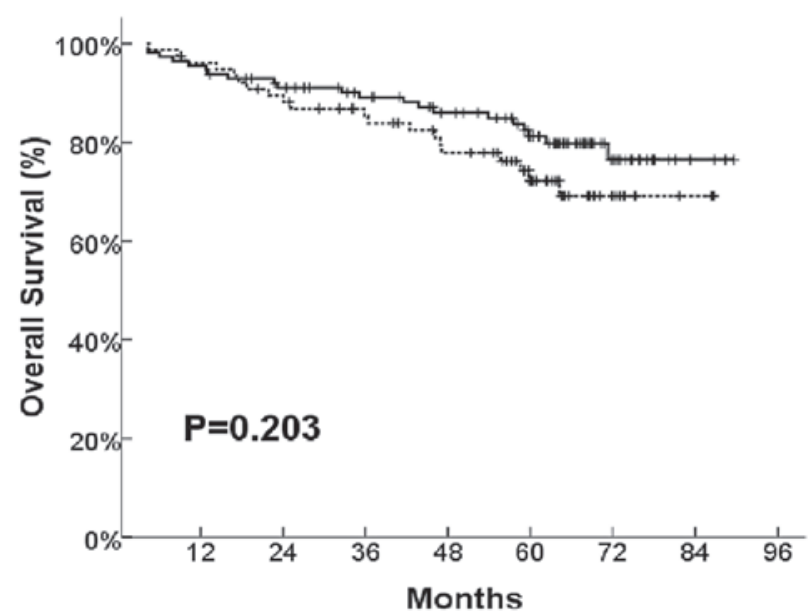

D

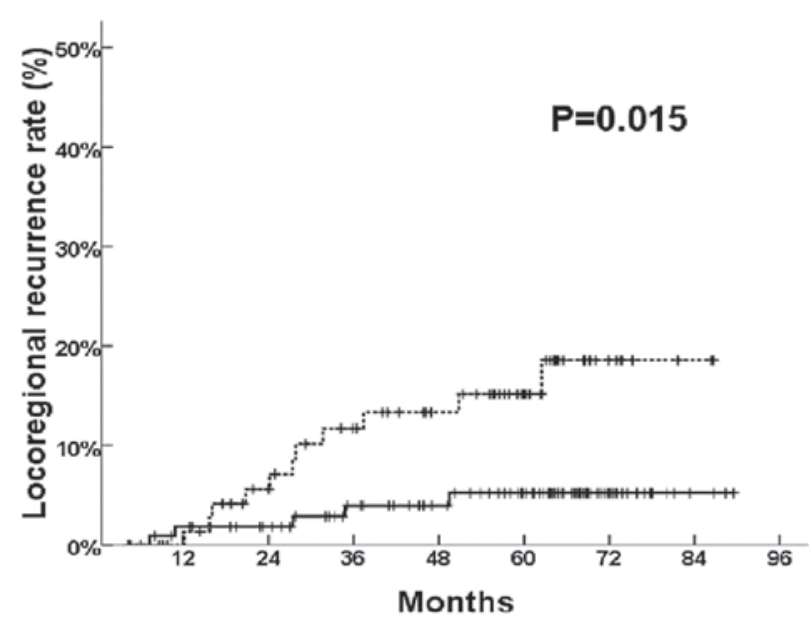

$\mathbf{F}$

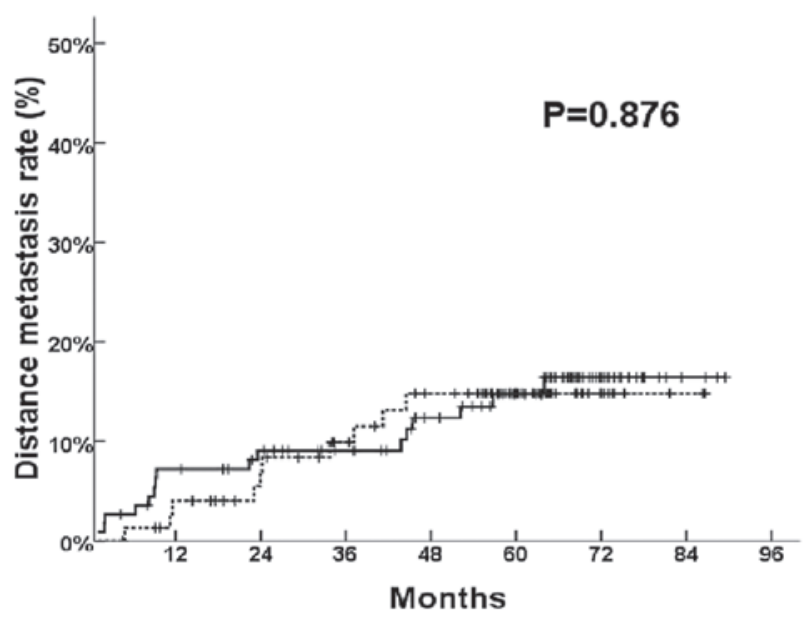

high-risk group

\section{................ Level lb unirradiated patients — Level Ib irradiated patients}

Figure 2. (A, C and E) Comparison of overall survival (OS), locoregional recurrence rate (LRR) and distant metastatic rate (DMR) between the level Ib irradiated and unirradiated patients in low-risk group. (B, D and F) Comparison of OS, LRR and DMR between the level Ib irradiated and unirradiated patients in high-risk group. 
In conclusion, the present study suggested a simple, practical and appropriate score model to predict the metastasis risk of level Ib lymph nodes. The factors included in the model were easily available in clinical practice. The model may be a valuable reference in CTV delineation of level Ib. More prospective future clinical studies are required to verify the results of the study.

\section{References}

1. Xia YF, Qian JY and Zhang EP: Practical Radiotherapy for Nasopharyngeal Carcinoma. PeKing University Medical Press, Beijing, pp86-87, 2003 (In Chinese).

2. Chen WZ, Gu BH, Chen HY, Gu ZY and Lin YG: Analysis of the results of treatment and the cases of failure following radiotherapy in 1083 cases of nasopharyngeal carcinoma. Ai Zheng 3: $160-162,1983$

3. Yi JL, Gao L, Huang XD, et al: Nasopharyngeal carcinoma treated by radical radiotherapy alone: Ten-year experience of a single institution. Int J Radiat Oncol Biol Phys 65: 161-168, 2006.

4. Su SF, Han F, Zhao C, et al: Treatment outcomes for different subgroups of nasopharyngeal carcinoma patients treated with intensity-modulated radiation therapy. Chin J Cancer 30: 565-573, 2011.

5. Grégoire V, Levendag P, Ang KK, et al: CT-based delineation of lymph node levels and related CTVs in the node-negative neck: DAHANCA, EORTC, GORTEC, NCIC, RTOG consensus guidelines. Radiother Oncol 69: 227-236, 2003.

6. Sun Y, Ma J, Lu TX, Wang Y, Huang Y and Tang LL: Regulation for distribution of metastatic cervical lymph nodes of 512 cases of nasopharyngeal carcinoma. Ai Zheng 23 (11 Suppl): 1523-1527, 2004 (In Chinese).

7. Wang XS, Hu CS, Wu YR, Qiu XX and Feng Y: Analysis of computed tomography-based distribution of metastatic cervical nodes in 218 cases of nasopharyngeal carcinoma. Ai Zheng 23: 1056-1059, 2004 (In Chinese).

8. Luo DH, Zhou CW, Yao XS and Zhao YF: The CT manifestations of the cervical lymph node metastasis of the nasopharyngeal carcinoma. J Clin Radiol 26: 1199-1203, 2007 (in Chinese).

9. Yeh SA, Tang Y, Lui CC, Huang YJ and Huang EY: Treatment outcomes and late complications of 849 patients with nasopharyngeal carcinoma treated with radiotherapy alone. Int J Radiat Oncol Biol Phys 62: 672-679, 2005.

10. Kuten A, Ben-Aryeh H,Berdicevsky I, et al: Oral side effects of head and neck irradiation: correlation between clinical manifestations and laboratory data. Int J Radiat Oncol Biol Phys 12: 401-405, 1986.

11. Jha N, Seikaly H, Harris J, et al: Prevention of radiation induced xerostomia by surgical transfer of submandibular salivary gland into the submental space. Radiother Oncol 66: 283-289, 2003.
12. Al-Qahtani K, Hier MP, Sultanum K and Black MJ: The role of submandibular salivary gland transfer in preventing xerostomia in the chemoradiotherapy patient. Oral Surg Oral Med Oral Pathol Oral Radiol Endod 101: 753-756, 2006.

13. Lee N, Xia P, Quivey JM, et al: Intensity-modulated radiotherapy in the treatment of nasopharyngeal carcinoma: an update of the UCSF experience. Int J Radiat Oncol Biol Phys 53: 12-22, 2002.

14. Kam MK, Teo PM, Chau RM, et al: Treatment of nasopharyngeal carcinoma with intensity-modulated radiotherapy: the Hong Kong experience. Int J Radiat Oncol Biol Phys 60: 1440-1450, 2004.

15. Liu WS, Kuo HC, Lin JC, et al: Assessment of salivary function change in nasopharyngeal carcinoma treated by parotid-sparing radiotherapy. Cancer J 12: 494-500, 2006.

16. Kam MK, Leung SF, Zee B, et al: Prospective randomized study of intensity-modulated radiotherapy on salivary gland function in early-stage nasopharyngeal carcinoma patients. J Clin Oncol 25: 4873-4879, 2007.

17. Tham IW, Hee SW, Yeo RM, et al: Treatment of nasopharyngeal carcinoma using intensity-modulated radiotherapy-the national cancer centre singapore experience. Int J Radiat Oncol Biol Phys 75: 1481-1486, 2009.

18. Radiation Therapy Oncology Group: RTOG 0615 Protocol Information. http://www.rtog.org/ClinicalTrials/ProtocolTable/ StudyDetails.aspx?study=0615. Accessed February 2, 2011.

19. International Union Against Cancer: TNM Classification of Malignant Tumors. Sobin LH, Gospodarowicz MK and Wittekind CH (eds). 7th edition. Wiley \& Sons, Inc., New York, NY, 2009.

20. van den Brekel MW, Stel HV, Castelijns JA, et al: Cervical lymph node metastasis: assessment of radiologic criteria. Radiology 177: 379-384, 1990.

21. Apfel CC, Läärä E, Koivuranta M, Greim CA and Roewer N: A simplified risk score for predicting postoperative nausea and vomiting: conclusions from cross-validations between two centers. Anesthesiology 91: 693-700, 1999.

22. Pan WR, Suami H, Corlett RJ and Ashton MW: Lymphatic drainage of the nasal fossae and nasopharynx: preliminary anatomical and radiological study with clinical implications. Head Neck 31: 52-57, 2009.

23. Zheng GL: Mode of spread in nasopharyngeal carcinoma as seen on CT scan. Zhonghua Zhong Liu Za Zhi 10: 293-295, 1988 (In Chinese).

24. King AD, Ahuja AT, Leung SF, et al: Neck node metastases from nasopharyngeal carcinoma: MR imaging of patterns of disease. Head Neck 22: 275-281, 2000.

25. van den Brekel MW, van der Waal I, Meijer CJ, Freeman JL, Castelijns JA and Snow GB: The incidence of micrometastases in neck dissection specimens obtained from elective neck dissections. Laryngoscope 106: 987-991, 1996. 